\title{
Symbolic aggregate approximation improves gap filling in high resolution mass spectrometry data processing
}

\section{Authors}

Erik Müller ${ }^{1,2,{ }^{*}}$, Carolin Elisabeth Huber ${ }^{1,2}$,Werner Brack ${ }^{1,2}$, Martin Krauss $^{1}$, Tobias Schulze ${ }^{1, *}$

\section{Affiliations}

1 UFZ - Helmholtz Centre for Environmental Research, Department of Effect-Directed Analysis, Permoserstraße 15, 04218 Leipzig, Germany

2 RWTH Aachen University, Institute for Environmental Research, Worringerweg 1, 52074 Aachen, Germany

Corresponding authors:

Erik Müller: erik.mueller@ufz.de

Tobias Schulze: tobias.schulze@ufz.de

\section{Table of Contents}
A. Results: .2

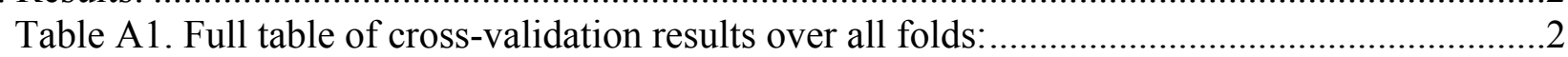
Graphic A1. Boxplot of the cross validation results across each fold and method ........................ 3
B. Sample and data processing information 


\section{A. Results:}

\section{Table A1. Full table of cross-validation results over all folds:}

\begin{tabular}{|c|c|c|c|c|c|c|c|c|c|}
\hline Accuracy & MCC & Sensitivity & Specificity & $\mathbf{a}$ & $\mathbf{w}$ & $\mathbf{t}$ & $\mathbf{p}$ & Fold & Method \\
\hline 0.86 & 0.57 & 0.49 & 0.97 & & & & & 1 & Full data set(No gaps filled) \\
\hline 0.87 & 0.54 & 0.48 & 0.97 & & & & & 2 & Full data set(No gaps filled) \\
\hline 0.87 & 0.58 & 0.53 & 0.96 & & & & & 3 & Full data set(No gaps filled) \\
\hline 0.85 & 0.54 & 0.48 & 0.96 & & & & & 4 & Full data set(No gaps filled) \\
\hline 0.88 & 0.59 & 0.54 & 0.97 & & & & & 5 & Full data set(No gaps filled) \\
\hline 0.88 & 0.57 & 0.52 & 0.97 & & & & & 6 & Full data set(No gaps filled) \\
\hline 0.86 & 0.56 & 0.49 & 0.97 & & & & & 7 & Full data set(No gaps filled) \\
\hline 0.87 & 0.57 & 0.5 & 0.97 & & & & & 8 & Full data set(No gaps filled) \\
\hline 0.88 & 0.63 & 0.56 & 0.97 & & & & & 9 & Full data set(No gaps filled) \\
\hline 0.87 & 0.58 & 0.51 & 0.97 & & & & & 10 & Full data set(No gaps filled) \\
\hline 0.47 & 0.3 & 1 & 0.31 & & & 3.5 & & 1 & Full data set (filled - trivial) \\
\hline 0.42 & 0.26 & 0.99 & 0.28 & & & 3.5 & & 2 & Full data set (filled - trivial) \\
\hline 0.45 & 0.29 & 1 & 0.3 & & & 3.5 & & 3 & Full data set (filled - trivial) \\
\hline 0.44 & 0.27 & 0.99 & 0.27 & & & 3.5 & & 4 & Full data set (filled - trivial) \\
\hline 0.43 & 0.27 & 1 & 0.28 & & & 3.5 & & 5 & Full data set (filled - trivial) \\
\hline 0.43 & 0.27 & 1 & 0.29 & & & 3.5 & & 6 & Full data set (filled - trivial) \\
\hline 0.47 & 0.29 & 0.99 & 0.31 & & & 3.5 & & 7 & Full data set (filled - trivial) \\
\hline 0.42 & 0.26 & 0.99 & 0.27 & & & 3.5 & & 8 & Full data set (filled - trivial) \\
\hline 0.46 & 0.29 & 0.99 & 0.31 & & & 3.5 & & 9 & Full data set (filled - trivial) \\
\hline 0.44 & 0.28 & 1 & 0.29 & & & 3.5 & & 10 & Full data set (filled - trivial) \\
\hline 0.61 & 0.33 & 0.86 & 0.53 & & & & & 1 & Full data set (filled - MZmine) \\
\hline 0.59 & 0.31 & 0.87 & 0.52 & & & & & 2 & Full data set (filled - MZmine) \\
\hline 0.58 & 0.31 & 0.87 & 0.51 & & & & & 3 & Full data set (filled - MZmine) \\
\hline 0.6 & 0.33 & 0.86 & 0.52 & & & & & 4 & Full data set (filled - MZmine) \\
\hline 0.59 & 0.31 & 0.86 & 0.52 & & & & & 5 & Full data set (filled - MZmine) \\
\hline 0.6 & 0.29 & 0.83 & 0.54 & & & & & 6 & Full data set (filled - MZmine) \\
\hline 0.63 & 0.35 & 0.86 & 0.56 & & & & & 7 & Full data set (filled - MZmine) \\
\hline 0.56 & 0.29 & 0.87 & 0.48 & & & & & 8 & Full data set (filled - MZmine) \\
\hline 0.63 & 0.35 & 0.86 & 0.56 & & & & & 9 & Full data set (filled - MZmine) \\
\hline 0.61 & 0.32 & 0.84 & 0.55 & & & & & 10 & Full data set (filled - MZmine) \\
\hline 0.87 & 0.66 & 0.79 & 0.9 & 6 & 11 & 7 & 0.9 & 1 & Full data set (filled - SAX) \\
\hline 0.86 & 0.6 & 0.76 & 0.88 & 6 & 11 & 7 & 0.9 & 2 & Full data set (filled - SAX) \\
\hline 0.87 & 0.67 & 0.86 & 0.88 & 8 & 8 & 14 & 0.9 & 3 & Full data set (filled - SAX) \\
\hline 0.87 & 0.67 & 0.79 & 0.9 & 6 & 11 & 7 & 0.9 & 4 & Full data set (filled - SAX) \\
\hline 0.88 & 0.66 & 0.79 & 0.9 & 6 & 11 & 7 & 0.9 & 5 & Full data set (filled - SAX) \\
\hline 0.86 & 0.65 & 0.88 & 0.86 & 8 & 8 & 14 & 0.9 & 6 & Full data set (filled - SAX) \\
\hline 0.86 & 0.65 & 0.84 & 0.87 & 8 & 8 & 14 & 0.9 & 7 & Full data set (filled - SAX) \\
\hline 0.88 & 0.65 & 0.79 & 0.9 & 6 & 11 & 7 & 0.9 & 8 & Full data set (filled - SAX) \\
\hline 0.87 & 0.68 & 0.86 & 0.88 & 8 & 8 & 14 & 0.9 & 9 & Full data set (filled - SAX) \\
\hline 0.88 & 0.66 & 0.75 & 0.92 & 6 & 11 & 7 & 0.9 & 10 & Full data set (filled - SAX) \\
\hline 0.41 & 0.24 & 1 & 0.32 & & & 3.5 & & 1 & Only gaps (filled - trivial) \\
\hline 0.37 & 0.2 & 0.98 & 0.29 & & & 3.5 & & 2 & Only gaps (filled - trivial) \\
\hline 0.39 & 0.22 & 0.99 & 0.31 & & & 3.5 & & 3 & Only gaps (filled - trivial) \\
\hline 0.38 & 0.22 & 0.99 & 0.28 & & & 3.5 & & 4 & Only gaps (filled - trivial) \\
\hline 0.37 & 0.2 & 0.99 & 0.29 & & & 3.5 & & 5 & Only gaps (filled - trivial) \\
\hline 0.38 & 0.21 & 1 & 0.3 & & & 3.5 & & 6 & Only gaps (filled - trivial) \\
\hline 0.41 & 0.23 & 0.98 & 0.32 & & & 3.5 & & 7 & Only gaps (filled - trivial) \\
\hline
\end{tabular}




\begin{tabular}{|c|c|c|c|c|c|c|c|c|c|}
\hline 0.36 & 0.2 & 0.99 & 0.28 & & & 3.5 & & 8 & Only gaps (filled - trivial) \\
\hline 0.4 & 0.21 & 0.98 & 0.32 & & & 3.5 & & 9 & Only gaps (filled - trivial) \\
\hline 0.38 & 0.22 & 1 & 0.3 & & & 3.5 & & 10 & Only gaps (filled - trivial) \\
\hline 0.57 & 0.19 & 0.72 & 0.55 & & & & & 1 & Only gaps (filled - MZmine) \\
\hline 0.56 & 0.18 & 0.74 & 0.53 & & & & & 2 & Only gaps (filled - MZmine) \\
\hline 0.55 & 0.16 & 0.71 & 0.53 & & & & & 3 & Only gaps (filled - MZmine) \\
\hline 0.57 & 0.2 & 0.74 & 0.54 & & & & & 4 & Only gaps (filled - MZmine) \\
\hline 0.55 & 0.15 & 0.7 & 0.54 & & & & & 5 & Only gaps (filled - MZmine) \\
\hline 0.57 & 0.13 & 0.65 & 0.56 & & & & & 6 & Only gaps (filled - MZmine) \\
\hline 0.59 & 0.2 & 0.72 & 0.57 & & & & & 7 & Only gaps (filled - MZmine) \\
\hline 0.52 & 0.15 & 0.73 & 0.5 & & & & & 8 & Only gaps (filled - MZmine) \\
\hline 0.59 & 0.17 & 0.68 & 0.58 & & & & & 9 & Only gaps (filled - MZmine) \\
\hline 0.58 & 0.15 & 0.67 & 0.56 & & & & & 10 & Only gaps (filled - MZmine) \\
\hline 0.87 & 0.53 & 0.69 & 0.9 & 8 & 8 & 14 & 0.9 & 1 & Only gaps (filled - SAX) \\
\hline 0.86 & 0.46 & 0.65 & 0.89 & 8 & 8 & 14 & 0.9 & 2 & Only gaps (filled - SAX) \\
\hline 0.88 & 0.53 & 0.7 & 0.91 & 8 & 8 & 14 & 0.9 & 3 & Only gaps (filled - SAX) \\
\hline 0.87 & 0.55 & 0.75 & 0.88 & 4 & 11 & 7 & 0.9 & 4 & Only gaps (filled - SAX) \\
\hline 0.87 & 0.5 & 0.73 & 0.89 & 4 & 11 & 7 & 0.9 & 5 & Only gaps (filled - SAX) \\
\hline 0.88 & 0.52 & 0.75 & 0.89 & 8 & 8 & 14 & 0.9 & 6 & Only gaps (filled - SAX) \\
\hline 0.87 & 0.51 & 0.68 & 0.89 & 8 & 8 & 14 & 0.9 & 7 & Only gaps (filled - SAX) \\
\hline 0.87 & 0.5 & 0.7 & 0.89 & 4 & 11 & 7 & 0.9 & 8 & Only gaps (filled - SAX) \\
\hline 0.88 & 0.49 & 0.66 & 0.91 & 4 & 11 & 7 & 0.7 & 9 & Only gaps (filled - SAX) \\
\hline 0.88 & 0.52 & 0.68 & 0.91 & 4 & 11 & 7 & 0.7 & 10 & Only gaps (filled - SAX) \\
\hline
\end{tabular}

\section{Graphic A2. Boxplot of the cross validation results across each fold and method}

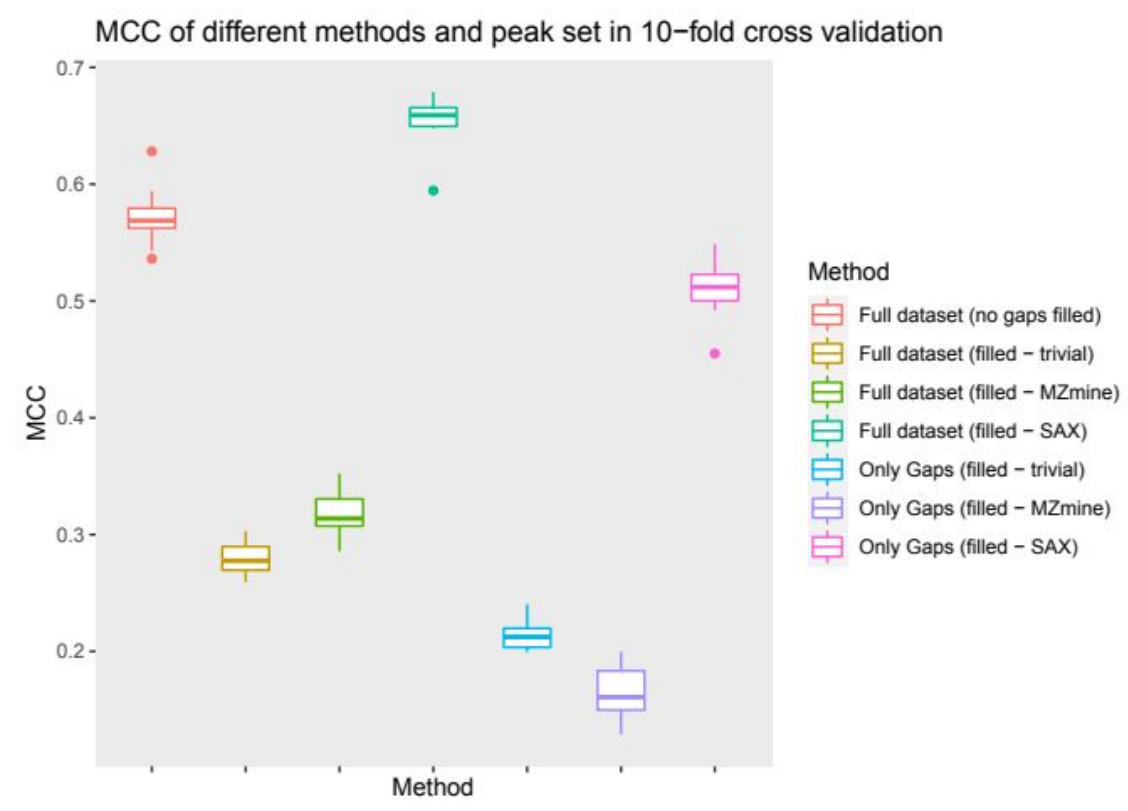

A2.: Boxplots representing the MCC for each fold of the 10-fold cross-validation of each of the seven method/dataset combinations: From left to right: $(1$, red): Utilizing the full data 
set but without any gap filling; (2, yellow): The results of the simple gap filling with the full data set; (3, green): The results of the Peak Finder algorithm with the whole data set; $(4$, turqoise): The results of the SAX gap filling with the whole data set; (5, blue): The results of the simple data set with evaluating only the gaps specifically; $(6$, violet): The results of the Peak Finder algorithm with evaluating only the gaps specifically; (7, pink): The results of the SAX gap filling with evaluating only the gaps specifically

\section{B. Sample and processing information}

The information in this part of the supplemental information has been taken from the Data Descriptor by Müller et al. ${ }^{1}$

\section{B1. Sample information}

The sampling was conducted at the Holtemme river (Saxony-Anhalt, Germany). The river originates in the Harz Mountains and flows into the Bode river after $47 \mathrm{~km}$. At 16 spots along the river, grab samples with a volume of $500 \mathrm{~mL}$ were taken. To guarantee relative consistency of these samples, the time at which they were taken was adjusted to the flow rate of the river to sample the same water package at all spots.

\section{B2. Sample information table}

\begin{tabular}{|c|c|c|c|c|c|}
\hline File Name & $\begin{array}{l}\text { Sequenc } 1 \\
\text { e No. }\end{array}$ & $\begin{array}{l}\text { Locatio Name of Location } \\
\mathrm{n} \text { No. }\end{array}$ & Latitude & Longitude & $\begin{array}{l}\text { Sample } \\
\text { type }\end{array}$ \\
\hline 161010_0_Blank.mzML & 0 & & & & Blank \\
\hline 161010_1_Blank.mzML & 1 & & & & Blank \\
\hline 161010_2_Cal_1ngL.mzML & 2 & & & & $\begin{array}{l}\text { Calibratio } \\
\mathrm{n}\end{array}$ \\
\hline 161010_3_Cal_10ngL.mzML & 3 & & & & $\begin{array}{l}\text { Calibratio } \\
\mathrm{n}\end{array}$ \\
\hline 161010_4_Cal_100ngL.mzML & 4 & & & & $\begin{array}{l}\text { Calibratio } \\
\mathrm{n}\end{array}$ \\
\hline 161010_5_Cal_1000ngL.mzML & 5 & & & & $\begin{array}{l}\text { Calibratio } \\
\mathrm{n}\end{array}$ \\
\hline 161010_6_Blank.mzML & 6 & & & & Blank \\
\hline 161010_7_Stich_5_T.mzML & 7 & 5Braunes Wasser & $\begin{array}{l}51^{\circ} 49^{\prime} 10.9^{\prime \prime} \\
\mathrm{N}\end{array}$ & $\begin{array}{l}10^{\circ} 44^{\prime} 29.9^{\prime \prime} \\
E\end{array}$ & $\begin{array}{l}\text { Secondar } \\
y\end{array}$ \\
\hline 161010_8_Stich_8_T.mzML & 8 & 8Zillierbach & $\begin{array}{l}51^{\circ} 50^{\prime} 01.0^{\prime \prime} \\
\mathrm{N}\end{array}$ & $\begin{array}{l}10^{\circ} 46^{\prime} 48.0^{\prime \prime} \\
E\end{array}$ & $\begin{array}{l}\text { Secondar } \\
y\end{array}$ \\
\hline 161010_9_Stich_12_T.mzML & 9 & 12Barrenbach & $\begin{array}{l}51^{\circ} 51^{\prime} 52.0^{\prime \prime} \\
N\end{array}$ & $\begin{array}{l}10^{\circ} 49^{\prime} 54.3^{\prime \prime} \\
E\end{array}$ & $\begin{array}{l}\text { Secondar } \\
y\end{array}$ \\
\hline 161010_10_Stich_14_T.mzML & 10 & 14Silstedter Bach & $\begin{array}{l}51^{\circ} 51^{\prime} 47.6^{\prime \prime} \\
N\end{array}$ & $\begin{array}{l}10^{\circ} 50^{\prime} 44.7^{\prime \prime} \\
E\end{array}$ & $\begin{array}{l}\text { Secondar } \\
y\end{array}$ \\
\hline 161010_11_Stich_19_T.mzML & 11 & 19Rothe & $\begin{array}{l}51^{\circ} 52^{\prime} 08.8^{\prime \prime} \\
N\end{array}$ & $\begin{array}{l}10^{\circ} 54^{\prime} 28.9^{\prime \prime} \\
E\end{array}$ & $\begin{array}{l}\text { Secondar } \\
y\end{array}$ \\
\hline 161010_12_Stich_20_T.mzML & 12 & $\begin{array}{l}\text { 20Hellbach/Muehlenb } \\
\mathrm{h}\end{array}$ & $\begin{array}{l}\mathrm{c} 51^{\circ} 52^{\prime} 32.1^{\prime \prime} \\
\mathrm{N}\end{array}$ & $\begin{array}{l}10^{\circ} 54^{\prime} 43.1^{\prime \prime} \\
E\end{array}$ & $\begin{array}{l}\text { Secondar } \\
\text { y }\end{array}$ \\
\hline 161010_13_Stich_23_T.mzML & 13 & 23Stroebecker Fliess & $\begin{array}{l}51^{\circ} 53^{\prime} 32.3^{\prime \prime} \\
\mathrm{N}\end{array}$ & $\begin{array}{l}10^{\circ} 58^{\prime} 36.5^{\prime \prime} \\
E\end{array}$ & $\begin{array}{l}\text { Secondar } \\
\text { y }\end{array}$ \\
\hline 161010_14_Stich_35_T.mzML & 14 & 35Asse & $\begin{array}{l}51^{\circ} 55^{\prime} 27.4^{\prime \prime} \\
\mathrm{N}\end{array}$ & $\begin{array}{l}11^{\circ} 06^{\prime} 16.6^{\prime \prime} \\
E\end{array}$ & $\begin{array}{l}\text { Secondar } \\
y\end{array}$ \\
\hline 161010_15_Stich_39_T.mzML & 15 & 39Ditch Nienhagen & $51^{\circ} 56^{\prime} 57.3^{\prime \prime}$ & $11^{\circ} 10^{\prime} 08.0^{\prime \prime}$ & Secondar \\
\hline
\end{tabular}




\begin{tabular}{|c|c|c|c|c|c|}
\hline & & & $\mathrm{N}$ & & $y$ \\
\hline 161010_16_Stich_41_T.mzML & 16 & 41Salzgraben & $\begin{array}{l}51^{\circ} 57^{\prime} 01.4^{\prime \prime} \\
\mathrm{N}\end{array}$ & $\begin{array}{l}11^{\circ} 10^{\prime} 38.7^{\prime \prime} \\
E\end{array}$ & $\begin{array}{l}\text { Secondar } \\
\text { y }\end{array}$ \\
\hline 161010_17_Blank.mzML & 17 & & & & Blank \\
\hline 161010_18_Blank.mzML & 18 & & & & Blank \\
\hline 161010_19_Stich_3.mzML & 19 & 3Mobicos Wernigerode & $\begin{array}{l}e 51^{\circ} 49^{\prime} 04.3^{\prime \prime} \\
N\end{array}$ & $\begin{array}{l}10^{\circ} 43^{\prime} 43.9^{\prime \prime} \\
\mathrm{E}\end{array}$ & Primary \\
\hline 161010_20_Stich_9.mzML & 20 & 9UPS RW drainage & $\begin{array}{l}51^{\circ} 50^{\prime} 49.7^{\prime \prime} \\
\mathrm{N}\end{array}$ & $\begin{array}{l}10^{\circ} 47^{\prime} 29.6^{\prime \prime} \\
\mathrm{E}\end{array}$ & Primary \\
\hline 161010_21_Stich_11.mzML & 21 & 11UPS Barrenbach & $\begin{array}{l}51^{\circ} 51^{\prime} 48.1^{\prime \prime} \\
N\end{array}$ & $\begin{array}{l}10^{\circ} 49^{\prime} 52.8^{\prime \prime} \\
E\end{array}$ & Primary \\
\hline 161010_22_Stich_13.mzML & 22 & 13UPS Silstedter Bach & $\begin{array}{l}51^{\circ} 51^{\prime} 53.4 " \\
N\end{array}$ & $\begin{array}{l}10^{\circ} 50^{\prime} 46.2^{\prime \prime} \\
E\end{array}$ & Primary \\
\hline 161010_23_Stich_15.mzML & 23 & 15UPS WWTP Silstedt & $\begin{array}{l}51^{\circ} 51^{\prime} 54.1^{\prime \prime} \\
N\end{array}$ & $\begin{array}{l}10^{\circ} 51 ' 31.0^{\prime \prime} \\
E\end{array}$ & Primary \\
\hline 161010_24_Stich_17.mzML & 24 & 17DS WWTP Silstedt & $\begin{array}{l}51^{\circ} 51^{\prime} 59.3 " \\
\mathrm{~N}\end{array}$ & $\begin{array}{l}10^{\circ} 52^{\prime} 47.0^{\prime \prime} \\
E\end{array}$ & Primary \\
\hline 161010_25_Blank.mzML & 25 & & & & Blank \\
\hline 161010_26_Stich_18.mzML & 26 & 18UPS Derenburg & $\begin{array}{l}51^{\circ} 52^{\prime} 00.4 " \\
\mathrm{~N}\end{array}$ & $\begin{array}{l}10^{\circ} 54^{\prime} 25.0^{\prime \prime} \\
E\end{array}$ & Primary \\
\hline 161010_27_Stich_21.mzML & 27 & 21DS Derenburg & $\begin{array}{l}51^{\circ} 52^{\prime} 37.5^{\prime \prime} \\
N\end{array}$ & $\begin{array}{l}10^{\circ} 54^{\prime} 46.7^{\prime \prime} \\
\mathrm{E}\end{array}$ & Primary \\
\hline 161010_28_Stich_22.mzML & 28 & 22Pegel Mahndorf & $\begin{array}{l}51^{\circ} 53^{\prime} 06.2^{\prime \prime} \\
N\end{array}$ & $\begin{array}{l}10^{\circ} 57^{\prime} 47.2^{\prime \prime} \\
E\end{array}$ & Primary \\
\hline 161010_31_Stich_26.mzML & 31 & $\begin{array}{l}\text { 26Halberstadt UPS RW } \\
\text { drainage }\end{array}$ & $\begin{array}{l}51^{\circ} 54^{\prime} 11.6^{\prime \prime} \\
\mathrm{N}\end{array}$ & $\begin{array}{l}11^{\circ} 03^{\prime} 28.2^{\prime \prime} \\
\mathrm{E}\end{array}$ & Primary \\
\hline 161010_32_Stich_31.mzML & 32 & $\begin{array}{l}\text { 31DS WWTP } \\
\text { Halberstadt }\end{array}$ & $\begin{array}{l}51^{\circ} 54^{\prime} 25.8^{\prime \prime} \\
\mathrm{N}\end{array}$ & $\begin{array}{l}11^{\circ} 04^{\prime} 12.6^{\prime \prime} \\
\mathrm{E}\end{array}$ & Primary \\
\hline 161010_33_Stich_34.mzML & 33 & 34UPS Asse & $\begin{array}{l}51^{\circ} 55^{\prime} 21.9^{\prime \prime} \\
\mathrm{N}\end{array}$ & $\begin{array}{l}11^{\circ} 06^{\prime} 25.8^{\prime \prime} \\
E\end{array}$ & Primary \\
\hline 161010_34_Stich_36a.mzML & $3436 a$ & $\begin{array}{l}\text { UPS Weir Gr. } \\
\text { Quenstedt }\end{array}$ & $\begin{array}{l}51^{\circ} 55^{\prime} 24.3^{\prime \prime} \\
\mathrm{N}\end{array}$ & $\begin{array}{l}11^{\circ} 06^{\prime} 36.3^{\prime \prime} \\
E\end{array}$ & Primary \\
\hline 161010_35_Blank.mzML & 35 & & & & Blank \\
\hline 161010_36_Stich_38.mzML & 36 & $\begin{array}{r}\text { 38MOBICOS } \\
\text { Nienhagen }\end{array}$ & $\begin{array}{l}51^{\circ} 56^{\prime} 29.7^{\prime \prime} \\
N\end{array}$ & $\begin{array}{l}11^{\circ} 09^{\prime} 31.1^{\prime \prime} \\
E\end{array}$ & Primary \\
\hline 161010_37_Stich_40.mzML & 37 & 40UPS Salzgraben & $\begin{array}{l}51^{\circ} 57^{\prime} 01.6^{\prime \prime} \\
\mathrm{N}\end{array}$ & $\begin{array}{l}11^{\circ} 10^{\prime} 33.4^{\prime \prime} \\
E\end{array}$ & Primary \\
\hline 161010_38_Stich_42.mzML & 38 & 42Mouth & $\begin{array}{l}51^{\circ} 57^{\prime} 47.8^{\prime \prime} \\
\mathrm{N}\end{array}$ & $\begin{array}{l}11^{\circ} 10^{\prime} 57.7^{\prime \prime} \\
\mathrm{E}\end{array}$ & Primary \\
\hline 161010_39_Stich_29_KA.mzML & 39 & 29WWTP Halberstadt & $\begin{array}{l}51^{\circ} 54^{\prime} 16.8^{\prime \prime} \\
\mathrm{N}\end{array}$ & $\begin{array}{l}11^{\circ} 03^{\prime} 47.1^{\prime \prime} \\
E\end{array}$ & Primary \\
\hline 161010_40_Blank.mzML & 40 & & & & Blank \\
\hline $\begin{array}{l}\text { 161010_41_KA_HBS_0900.mz } \\
\text { ML }\end{array}$ & 41 & 29WWTP Halberstadt & $\begin{array}{l}51^{\circ} 54^{\prime} 16.8^{\prime \prime} \\
\mathrm{N}\end{array}$ & $\begin{array}{l}11^{\circ} 03^{\prime} 47.1^{\prime \prime} \\
\mathrm{E}\end{array}$ & $\begin{array}{l}\text { Secondar } \\
y\end{array}$ \\
\hline $\begin{array}{l}\text { 161010_42_KA_HBS_1100.mz } \\
\text { ML }\end{array}$ & 42 & 29WWTP Halberstadt & $\begin{array}{l}51^{\circ} 54^{\prime} 16.8^{\prime \prime} \\
\mathrm{N}\end{array}$ & $\begin{array}{l}11^{\circ} 03^{\prime} 47.1^{\prime \prime} \\
E\end{array}$ & $\begin{array}{l}\text { Secondar } \\
y\end{array}$ \\
\hline $\begin{array}{l}\text { 161010_43_KA_HBS_1300.mz } \\
\text { ML }\end{array}$ & 43 & 29WWTP Halberstadt & $\begin{array}{l}51^{\circ} 54^{\prime} 16.8^{\prime \prime} \\
\mathrm{N}\end{array}$ & $\begin{array}{l}11^{\circ} 03^{\prime} 47.1^{\prime \prime} \\
\mathrm{E}\end{array}$ & $\begin{array}{l}\text { Secondar } \\
y\end{array}$ \\
\hline 161010_44_Blank.mzML & 44 & & & & Blank \\
\hline 161010_45_KA_SIL_0730.mzM & 45 & 16WWTP Silstedt & $\begin{array}{l}51^{\circ} 51^{\prime} 59.4 " \\
N\end{array}$ & $\begin{array}{l}10^{\circ} 51^{\prime} 43.3^{\prime \prime} \\
E\end{array}$ & $\begin{array}{l}\text { Secondar } \\
y\end{array}$ \\
\hline $\begin{array}{l}\text { 161010_46_KA_SIL_0930.mzM } \\
\text { L }\end{array}$ & 46 & 16WWTP Silstedt & $\begin{array}{l}51^{\circ} 51^{\prime} 59.4 " \\
N\end{array}$ & $\begin{array}{l}10^{\circ} 51^{\prime} 43.3^{\prime \prime} \\
E\end{array}$ & $\begin{array}{l}\text { Secondar } \\
y\end{array}$ \\
\hline $\begin{array}{l}\text { 161010_47_KA_SIL_1130.mzM } \\
\text { L }\end{array}$ & 47 & 16WWTP Silstedt & $\begin{array}{l}51^{\circ} 51^{\prime} 59.4 " \\
\mathrm{~N}\end{array}$ & $\begin{array}{l}10^{\circ} 51^{\prime} 43.3^{\prime \prime} \\
E\end{array}$ & $\begin{array}{l}\text { Secondar } \\
y\end{array}$ \\
\hline 161010_48_Blank.mzML & 48 & & & & Blank \\
\hline 161010_49_Cal_1ngL.mzML & 49 & & & & $\begin{array}{l}\text { Calibratio } \\
\mathrm{n}\end{array}$ \\
\hline 161010_50_Cal_10ngL.mzML & 50 & & & & $\begin{array}{l}\text { Calibratio } \\
\mathrm{n}\end{array}$ \\
\hline
\end{tabular}




\section{B3. Chemical analysis}

The analysis was performed on an UltiMate 3000 LC system (Thermo Scientific) connected to a QExactive Plus mass spectrometer (Thermo Scientific) with a HESI source. To prepare the samples for a direct large volume injection of $100 \mu \mathrm{L}$, a $2 \mathrm{M}$ ammonium formate buffer, $25 \mu \mathrm{L}$ of methanol and $25 \mu \mathrm{L}$ of an internal standard mixture containing 40 isotope-labelled compounds $(40 \mathrm{ng} / \mathrm{mL})$ were added to $1 \mathrm{~mL}$ of sample. These 40 internal standards were added to all measured samples except for the blanks.

A Kinetex $2.6 \mu \mathrm{m}$ EVO C18 $(50 \times 2.1 \mathrm{~mm})$ column with a pre-column (C18 EVO $5 \times 2.1$ $\mathrm{mm}$ ) and an inline filter was used at $40^{\circ} \mathrm{C}$ to perform chromatographic separation of the samples. The solvent gradient across all samples was identical.

The data was measured at the nominal resolving power of 140,000 referenced to $\mathrm{m} / \mathrm{z} 200$ in full-scan mode with a positive ionization. The sheath gas flow rate was 45 , the aux gas flow rate was 1 . The spray voltage was set to $3.8 \mathrm{kV}$. The capillary temperature and the aux gas heater temperature were both set to $300^{\circ} \mathrm{C}$. The S-lens RF level was 70 . The scan range was set to a m/z of 100-1000. At the beginning and at the end of the batch, calibration standards were run at $1,10,100$, and $1000 \mathrm{ng} / \mathrm{L}$ for quality control purposes.

\section{B4. Data processing}

Raw data from the LC-HRMS were converted into mzML format (centroid mode) by ProteoWizard v3.0.18265. Peak lists were generated using the software MZmine v2.26 with the following settings: 


\section{Mass Detection}
(a) Noise level $5 \mathrm{e} 3$

\section{ADAP chromatogram builder}
(a) Min group size of \# of scans: 15
(b) Group intensity threshold: 5000
(c) Min highest intensity: 5000
(d) $\mathrm{m} / \mathrm{z}$ tolerance: $0.001 \mathrm{~m} / \mathrm{z}$ or $7 \mathrm{ppm}$

\section{Smoothing}

(a) Filter width: 7

\section{Chromatogram deconvolution}

(a) Algorithm: Local minimum search

(b) Chromatographic threshold: $80.0 \%$

(c) Search minimum in RT range (min): 0.15

(d) Minimum relative height: $20.0 \%$

(e) Minimum absolute height: $5.0 \mathrm{e} 4$

(f) Min ratio of peak top/edge: 2.7

(g) Peak duration range ( $\mathrm{min}): 0.15$ - 4.00 - Range of acceptable peak durations

\section{Alignment}
(a) $\mathrm{m} / \mathrm{z}$ tolerance: 0.001 or $7 \mathrm{ppm}$
(b) Weight for $\mathrm{m} / \mathrm{z}$ : 80
(c) Retention time tolerance: 0.3 absolute (min)
(d) Weight for RT: 30 


\section{Bibliography}

(1) Müller, E.; Huber, C.; Beckers, L.-M.; Brack, W.; Krauss, M.; Schulze, T. A Data Set of 255,000 Randomly Selected and Manually Classifed Extracted Ion Chromatograms for Evaluation of Peak Detection Methods. Metabolites 2020, 10. 